Article

\title{
Thermal Analysis of Crystallization and Phase Transition in Novel Polyethylene Glycol Grafted Butene-1 Copolymers
}

\author{
Chuanbin An ${ }^{1}$, Yulian Li ${ }^{1}$, Yahui Lou ${ }^{1}$, Dongpo Song ${ }^{1}$, Bin Wang ${ }^{1}$, Li Pan ${ }^{1} \oplus$, Zhe Ma ${ }^{1, *}$ and \\ Yuesheng $\mathrm{Li}^{1,2}$ \\ 1 Tianjin Key Laboratory of Composite and Functional Materials, and School of Materials Science and \\ Engineering, Tianjin University, Tianjin 300072, China; ancb0306@tju.edu.cn (C.A.); \\ liyulian@tju.edu.cn (Y.L.); yhlou@tju.edu.cn (Y.L.); dongpo.song@tju.edu.cn (D.S.); \\ binwang@tju.edu.cn (B.W.); lilypan@tju.edu.cn (L.P.); ysli@tju.edu.cn (Y.L.) \\ 2 Collaborative Innovation Center of Chemical Science and Engineering (Tianjin), Tianjin 300072, China \\ * Correspondence: zhe.ma@tju.edu.cn; Tel.: +86-22-2740-2887
}

Received: 14 March 2019; Accepted: 6 May 2019; Published: 8 May 2019

\begin{abstract}
Copolymerization is an effective strategy to regulate the molecular structure and tune crystalline structures. In this work, novel butene-1 copolymers with different polyethylene glycol (PEG) grafts (number-average molecular weight $M_{\mathrm{n}}=750,2000$, and $4000 \mathrm{~g} / \mathrm{mol}$ ) were synthesized, for the first time introducing long-chain grafts to the polybutene-1 main chain. For these PEG-grafted copolymers, crystallization, melting, and phase transition behaviors were explored using differential scanning calorimetry. With respect to the linear homopolymer, the incorporation of a trimethylsilyl group decreases the cooling crystallization temperature $\left(T_{\mathrm{c}}\right)$, whereas the presence of the long PEG grafts unexpectedly elevates $T_{\mathrm{c}}$. For isothermal crystallization, a critical temperature was found at $70{ }^{\circ} \mathrm{C}$, below which all polyethylene glycol-grafted butene-1 (PB-PEG) copolymers have faster crystallization kinetics than polybutene-1 (PB). The subsequent melting process shows that for the identical crystallization temperature, generated PB-PEG crystallites always have lower melting temperatures than that of PB. Moreover, the II-I phase transition behavior of copolymers is also dependent on the length of PEG grafts. When form II, obtained from isothermal crystallization at $60{ }^{\circ} \mathrm{C}$, was annealed at $25^{\circ} \mathrm{C}$, PB-PEG-750, with the shortest PEG grafts of $M_{\mathrm{n}}=750 \mathrm{~g} / \mathrm{mol}$, could have the faster transition rate than PB. However, PB-PEG-750 exhibits a negative correlation between transition rate and crystallization temperature. Differently, in PB-PEG copolymers with PEG grafts $M_{\mathrm{n}}=2000$ and $4000 \mathrm{~g} / \mathrm{mol}$, transition rates rise with elevating crystallization temperature, which is similar with homopolymer PB. Therefore, the grafting of the PEG side chain provides the available method to tune phase transition without sacrificing crystallization capability in butene- 1 copolymers.
\end{abstract}

Keywords: butene-1 copolymer; long-chain graft; crystallization kinetics; melting; phase transition

\section{Introduction}

Polybutene-1 (PB-1) is one class of polymeric material which has outstanding mechanical properties, such as excellent creep resistance, good crack resistance, good heat resistance, and high hardness [1-8]. There are three possible crystal modifications in PB-1, which are the hexagonal form I/I', tetragonal form II, and orthorhombic form III [9-11]. From the thermodynamic point of view, the hexagonal phase is most stable, of which form I' is obtained directly by melt or solution crystallization, and form I is converted from the metastable tetragonal form II through solid-solid phase transformation [12-14]. Although tetragonal form II is metastable, it is the crystal modification that is usually generated from melt crystallization due to the kinetic advantage $[15,16]$. In this case, a phase transformation 
spontaneously happens from the initially generated form (form II) into the stable form (form I), even under quiescent conditions at room temperature $[2,17,18]$. The crystallites affected by such a phase transition are of great importance to product properties. On one hand, after II-I phase transition, the melting temperature is elevated and the mechanical strength of hardness is improved. On the other hand, when transformation is completely suppressed, form II exhibits a very high fracture strain of over $1000 \%$ [19]. Therefore, control of crystallite modification is crucial to material performance $[2,5,20]$.

In addition to the physical means to tune the rate of phase transition [1,21-27], the chemical regulation of macromolecular structure is another efficient method [28-32]. The molecular structure can be designed by varying molecular weight, as well as regularity, or incorporating the extra structural co-units. Recently, Qiao et al. studied the correlation of the II-I phase transition with molecular weights in a polybutene- 1 homopolymer [12,33,34]. It was found that high molecular weight facilitates the formation of the intercrystallite links because of the relative large radius of gyration, with respect to the stacking size of crystalline and amorphous domains, which enhances stress transfer into the lamellae. De Rosa et al. studied the effect of regularity on II-I phase transition [35,36]. Their results demonstrated that $r r$ triad defects can significantly promote the phase transition rate and even induce the appearance of hexagonal form I' directly from amorphous melt.

Copolymerization is also widely used to regulate molecular structure due to the diversity of comonomer types. In the 1960s, Jones studied the influence of $\alpha$-olefins with no more than 18 carbon atoms on the phase transition of copolymers [31]. It was found that the relatively short comonomers, including ethylene, propylene, and pentene, were able to accelerate phase transition, and the relatively long $\alpha$-olefin, with $6-18$ carbon atoms, slows down phase transition. Zheng et al. incorporated the branched 4-methyl-1-pentene (4M1P) co-units into the polybutene-1 main chain and found that $7.8 \mathrm{~mol} \%$ 4M1P co-units can completely suppress the occurrence of the II-I phase transition for their experimental duration of four months [19]. Moreover, He et al. utilized 1,5-hexadiene as the comonomer and reported that the incorporated ring-like structural units can also accelerate phase transition [37].

However, the presence of extra co-units may reduce crystallization capability due to the disturbed regularity of the main chain. For example, in the above butene-1/4M1P copolymers, the increase of 4M1P co-units from 0.18 to $12.3 \mathrm{~mol} \%$ significantly decreases the crystallization temperature from 73.4 to $25.5^{\circ} \mathrm{C}$, with cooling at $5{ }^{\circ} \mathrm{C} / \mathrm{min}$ [19]. For this problem, a long-chain branch (LCB) provides a potential solution [38-42]. Takamura et al. reported that the introduction of a LCB can significantly accelerate crystallization kinetics [38]. Wang et al. recently prepared a series of long-chain branched polylactide (PLA) and found that, with respect to linear PLA, the presence of LCB increases the nucleation density but reduces the linear growth rate [40]. Inspired by these studies, we wonder whether such long branches can be used in butene- 1 copolymers to tune phase transition without compromising the crystallization of the polymer. In actuality, Tarallo et al. studied the crystallization of butene- $1 /$ hexene copolymers. Their results show that the increase of hexene concentration from 3.3 to $11.2 \mathrm{~mol} \%$ decreases the crystallization temperature by more than $20^{\circ} \mathrm{C}$ [28]. Apparently, this conjecture does not work for the short branches like hexane. For longer grafts, the specific influences on PB-1 crystallization and phase transition remain mysterious.

In this work, we aim to explore the correlations between relatively long grafts with crystallization and phase transition behaviors. With the reaction intermediate method, long polyethylene glycol (PEG) grafts were successfully introduced to a polybutene- 1 backbone. Three PEG grafts, with number-average molecular weights of $M_{n}=750,2000$, and $4000 \mathrm{~g} / \mathrm{mol}$, respectively, were utilized to vary the length grafts, which kept the graft density identical. For these model copolymers, we explored the (non-isothermal and isothermal) crystallization and melting behaviors. Moreover, we also report that variation of the PEG graft length is an effective approach to tune the phase transition from tetragonal form II to hexagonal form I for polybutene-1 copolymers. 


\section{Materials and Methods}

\subsection{Materials}

All synthetic experiments involving air- and/or moisture-sensitive materials were conducted in an MBraun glovebox or by using the standard Schlenk technique under a nitrogen atmosphere. The $\left\{\mathrm{Me}_{2} \mathrm{Si}\left(2,5-\mathrm{Me}_{2}-3-(2-\mathrm{MePh})\right.\right.$ cyclopento[2,3-b]thiophen-6-yl) 2$\} \mathrm{ZrCl}_{2}$ (rac:meso = 1:1) precatalyst and poly (ethylene glycol) azide at one end (PEG-N 3 ) were synthesized according to the literature [43-45]. Toluene was used after purification by an Etelux solvent treatment system. The butene- 1 gas and ultra-high-purity nitrogen were purchased from the Tianjin Liufang Industrial Gas Distribution Co., Ltd. The triisobutyl aluminum $\left(\mathrm{Al}\left({ }^{i} \mathrm{Bu}\right)_{3}, 1.1 \mathrm{~mol} / \mathrm{L}\right.$ in toluene), trityltetrakis(pentauorophenyl)borate $\left(\left[\mathrm{Ph}_{3} \mathrm{C}\right]\left[\mathrm{B}\left(\mathrm{C}_{6} \mathrm{~F}_{5}\right)_{4}\right]\right)$ cocatalyst, cuprous bromide $(\mathrm{CuBr})$, pentamethyldiethylenetriamine (PMEDTA), and tetrabutylammonium fluoride (TBAF) were obtained from J\&K China Chemical Ltd. (Beijing, China) and were used directly without further purification. The synthetic route of the polybutene-1 copolymer and PEG-grafted polybutene-1 is shown in Scheme 1.

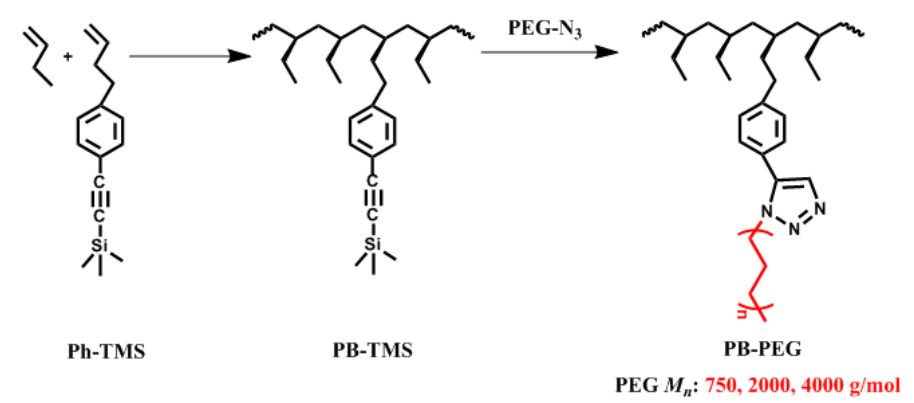

Scheme 1. Synthetic route of butene-1/4-[4-(trimethylsilylalkynyl)-phenyl]-1-butylene (Ph-TMS) copolymer and polyethylene glycol (PEG)-grafted butene-1 copolymers.

\subsection{Copolymerization of Butene-1 with Ph-TMS}

The process of the polymerization reaction is strictly required to be water and air free. The whole reaction system had a capacity of $90 \mathrm{~mL}$. The butene- 1 gas (ambient pressure) was fed into the Schlenk flask $(150 \mathrm{~mL})$ while the comonomer $(0.4 \mathrm{~mL})$ and $\mathrm{Al}\left({ }^{\mathrm{i}} \mathrm{Bu}\right)_{3}(1.5 \mathrm{~mL})$ were carefully injected into the system. Then, an appropriate amount of toluene was added into the flask to maintain the entire system volume at $70 \mathrm{~mL}$. Next, the solution was mechanical stirred at $400 \mathrm{rpm}$ for $5 \mathrm{~min}$ at room temperature to ensure that the butene- 1 formed a saturated solution in the presence of toluene. The catalyst $(15 \mu \mathrm{mol})$ and cocatalyst $(30 \mu \mathrm{mol})$ were dissolved separately with $20 \mathrm{~mL}$ of toluene and rapidly added into the system. Immediately, the rotation speed was increased to $900 \mathrm{rpm}$ and kept constant for 6 min. The pressure of the butene- 1 gas throughout the reaction process was maintained using a rubber balloon. Copolymerization was terminated by removing the source of butene- 1 gas and injecting $2 \mathrm{~mL}$ of $\mathrm{MeOH}$. The reaction solution was poured into an appropriate amount of ethanol to afford the precipitation of the copolymer. This copolymer was re-dissolved in toluene and precipitated with large amounts of ethanol to remove the unreacted monomer and residual catalyst. Finally, the butene-1/Ph-TMS (PB-TMS) copolymer was obtained by drying in a vacuum oven at $40{ }^{\circ} \mathrm{C}$ for $24 \mathrm{~h}$ to a constant weight (15.4 g). NMR spectra confirmed the successful synthesis of the copolymer (Figure S1a).

\subsection{Synthesis of Polybutene-1 Grafted with Polyethylene Glycol}

The grafted polybutene-1, with different molecular weights of PEG, was prepared via a process which requires strict control of the anhydrous and oxygen-free environment. Grafting experiments of PEG with different molecular weights were carried out in a similar manner. The experiment is described by taking PEG with a molecular weight of $750 \mathrm{~g} / \mathrm{mol}$ as an example. The copolymer (1.43 g) was dissolved in ultra-dry toluene $(70 \mathrm{~mL})$ at $70{ }^{\circ} \mathrm{C}$. A trace amount of TBAF $(9 \mathrm{~mol} \%$ of the total monomer content, $0.02 \mathrm{~mL}$ ) was placed in the reactor as a catalyst to remove trimethylsilyl groups. 
After $6 \mathrm{~h}$ of reaction, PEG-N $\mathrm{N}_{3}(0.262 \mathrm{~g}), \mathrm{CuBr}(0.0245 \mathrm{mg})$ and PMEDTA $(0.21 \mathrm{~mL})$ were added to the reaction system in sequence. After reacting at $70{ }^{\circ} \mathrm{C}$ for $12 \mathrm{~h}$, the polymer was precipitated in cold n-hexane. The obtained polymer was washed with acetone three times. The final PEG-grafted polybutene-1 (1.52 g) was obtained after drying in vacuum at $40^{\circ} \mathrm{C}$ for $24 \mathrm{~h}$. NMR results proved that the grafting reaction was complete (Figure $\mathrm{S} 1 \mathrm{~b}$ ).

\subsection{Methods}

2.4.1. Nuclear Magnetic Resonance $\left({ }^{1} \mathrm{H}-\mathrm{NMR}\right)$ and Gel Permeation Chromatography (GPC) Characterization

The ${ }^{1} \mathrm{H}-\mathrm{NMR}$ characterizations were performed with a Bruker $400 \mathrm{MHz}$ spectrometer. All samples were tested at $120{ }^{\circ} \mathrm{C}$ with $\mathrm{C}_{2} \mathrm{D}_{2} \mathrm{Cl}_{4}$ as a solvent. From the ${ }^{1} \mathrm{H}-\mathrm{NMR}$ results, the Ph-TMS co-unit incorporations were determined using the formula Ph-TMS mol\% $=\left[8 I_{7.39-7.51 \mathrm{ppm}} /\left(2 I_{0.58-1.83 \mathrm{ppm}}-\right.\right.$ $\left.\left.7 I_{7.39-7.51} \mathrm{ppm}\right)\right] \times 100 \%$, where $I_{\mathrm{xppm}}$ is the peak integral of a proton at a certain $(\mathrm{x}) \mathrm{ppm}$. The basic molecular information, including the molecular weights and molecular weight distributions, was obtained at $150^{\circ} \mathrm{C}$ using a PL-GPC 220 high-temperature gel permeation chromatography (Figure S2). The measured molecular characteristics of the polybutene- 1 homopolymer, butene- $1 / \mathrm{Ph}-\mathrm{TMS}$ copolymer, and PEG-grafted polybutene- 1 are summarized in Table 1.

Table 1. Molecular characteristics of the homopolymer, copolymer, and PEG-grafted copolymers.

\begin{tabular}{|c|c|c|c|c|}
\hline Sample Code & Incorp. ${ }^{a}$ (mol \%) & $M_{n}$ of PEG (Da) & $M_{\mathrm{w}}^{\mathrm{b}}\left(10^{5} \mathrm{Da}\right)$ & $M_{\mathrm{w}} / M_{\mathrm{n}}{ }^{\mathrm{b}}$ \\
\hline PB & 0 & None & 1.98 & 2.38 \\
\hline PB-TMS & & None & 2.08 & 2.84 \\
\hline PB-PEG-750 & & 750 & 2.59 & 2.50 \\
\hline PB-PEG-2000 & 0.49 & 2000 & 2.77 & 2.44 \\
\hline PB-PEG-4000 & & 4000 & 3.05 & 2.93 \\
\hline
\end{tabular}

a Comonomer incorporation (mol\%) was determined by ${ }^{1} \mathrm{H}-\mathrm{NMR}$ spectra at $120{ }^{\circ} \mathrm{C}$ with $\mathrm{C}_{2} \mathrm{D}_{2} \mathrm{Cl}_{4}$ as a solvent.

b Determined by gel permeation chromatography (GPC) at $150{ }^{\circ} \mathrm{C}$ in $1,2,4-\mathrm{C}_{6} \mathrm{Cl}_{3} \mathrm{H}_{3}$ versus polystyrene standards.

\subsubsection{Differential Scanning Calorimetry (DSC)}

Differential scanning calorimetry (DSC) measurements were carried out with DSC Q2000 (TA instrument) under a nitrogen atmosphere. Three thermal protocols were employed in this work, in which the sample was first annealed at $180{ }^{\circ} \mathrm{C}$ for $10 \mathrm{~min}$ to clean the thermal history. Then, the crystallization ability was examined by dynamic cooling experiments at $10^{\circ} \mathrm{C} / \mathrm{min}$ (Figure S3a). In addition, the isothermal crystallizations were performed at various temperatures to reveal the correlation between crystallization kinetics and molecular structures (Figure S3b).

To explore the II-I phase transition, form II was prepared by isothermal crystallization at different temperatures, which was followed by $50 \mathrm{~h}$ annealing at $25^{\circ} \mathrm{C}$ (Figure S3c). At last, a heating process was applied at $10^{\circ} \mathrm{C} / \mathrm{min}$ to examine the generated crystallites.

\subsubsection{Fourier Transform Infrared Spectroscopy (FTIR)}

A Nicolet 6700 Fourier transform infrared (FTIR) spectrometer with a resolution of $4 \mathrm{~cm}^{-1}$ was employed to characterize the modification of the PB-1 crystallites. For the FTIR experiments, the samples were first compressed into thin films of around $200 \mu \mathrm{m}$. Then, a Linkam hot stage was used to prepare the crystallites, employing the same thermal protocol as with the DSC measurements. 


\section{Results and Discussion}

\subsection{Non-Isothermal Crystallization}

First of all, the effects of TMS branch and PEG graft on copolymer crystallization were investigated with the DSC dynamic cooling protocol, illustrated by Figure S3a. From the results of Figure 1, it can be seen that due to the disturbed chain regularity, the incorporation of a TMS branch lowers the crystallization temperature $\left(T_{\mathrm{c}}\right)$ from $58.3^{\circ} \mathrm{C}$ in linear $\mathrm{PB}$ to $36.5^{\circ} \mathrm{C}$ in the PB-TMS copolymer. However, it is interesting to observe that when the TMS branch was replaced by the long PEG grafts, $T_{\mathrm{C}}$ was elevated significantly, although the PB-TMS and PB-PEG copolymers have the same branch density. Moreover, the enhancement amplitude of crystallization by PEG is so high that the crystallization temperature of the PB-PEG copolymers becomes higher than that of PB. Table 2 summarizes the values of $T_{\mathrm{C}}$ as a function of PEG length. Clearly, PB-PEG-750 has the highest crystallization temperature of $68.1^{\circ} \mathrm{C}$, which is around $32{ }^{\circ} \mathrm{C}$ higher than PB-TMS. As the length of PEG graft was increased in PB-PEG-2000 and PB-PEG-4000, the crystallization temperatures are slightly decreased to around $63{ }^{\circ} \mathrm{C}$, which is still higher than the copolymer PB-TMS. It can be concluded that long PEG grafts with $M_{\mathrm{n}}$ between 750 and $4000 \mathrm{~g} / \mathrm{mol}$ have a distinct influence in elevating the cooling crystallization temperature with respect to PB-TMS.

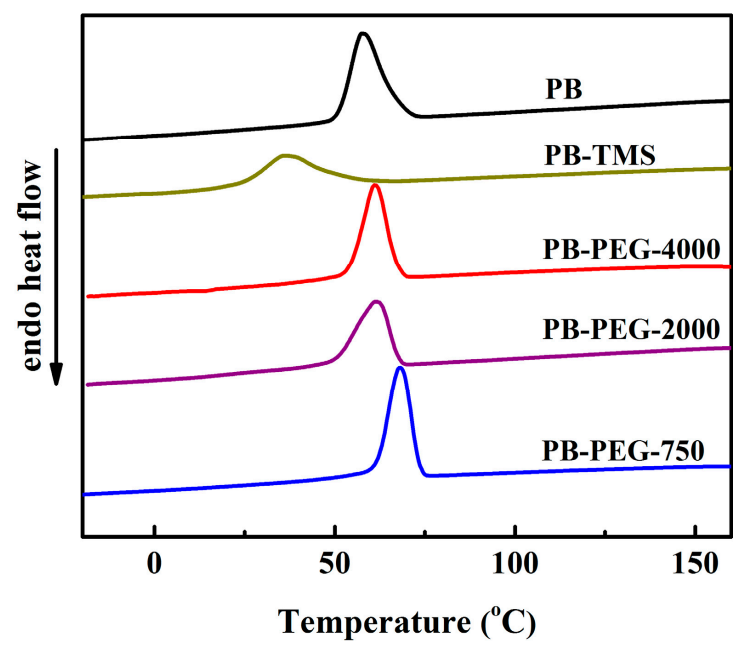

Figure 1. Differential scanning calorimetry (DSC) cooling curves of the homopolymer polybutene-1 (PB), copolymer PB-TMS, and polyethylene glycol (PEG)-grafted butene-1 copolymers.

Table 2. Crystallization temperature $\left(T_{c}\right)$ and crystallinity $\left(X_{C}\right)$ of the PB, PB-TMS, and PB-PEG copolymers.

\begin{tabular}{cccccc}
\hline Sample Code & PB & PB-TMS & PB-PEG-4000 & PB-PEG-2000 & PB-PEG-750 \\
\hline$T_{\mathrm{c}}\left({ }^{\circ} \mathrm{C}\right)$ & 58.3 & 36.5 & 63.2 & 63.4 & 68.1 \\
$X_{\mathrm{c}}(\%)$ & 41.0 & 29.3 & 39.1 & 38.5 & 39.1 \\
\hline
\end{tabular}

It has been reported that the copolymerization of structural co-units to the polybutene- 1 main chain may vary the crystallization polymorphism from the regular form (tetragonal form II) to the thermodynamically stable hexagonal form I' $[37,46]$. It is also known that the former has a much faster growth rate than the latter [47]. Then, concerning the aforementioned increased $T_{\mathrm{c}}$ in PEG-grafted copolymers, the modification of cooling-generated crystallites should be examined to know whether the contribution arises from the variation of crystallization polymorphism. Figure 2 shows the FTIR results for the PB, PB-TMS and PB-PEG copolymers after cooling to room temperature. Clearly, a single absorption band is observed at the wavenumber of $904 \mathrm{~cm}^{-1}$, which corresponds to tetragonal form II [48]. There is no signal identified at $925 \mathrm{~cm}^{-1}$, which is the characteristic band for polybutene-1 
hexagonal modification [48]. Then, it can be concluded that variation of crystallization polymorphism does not contribute to above affected crystallization temperature.

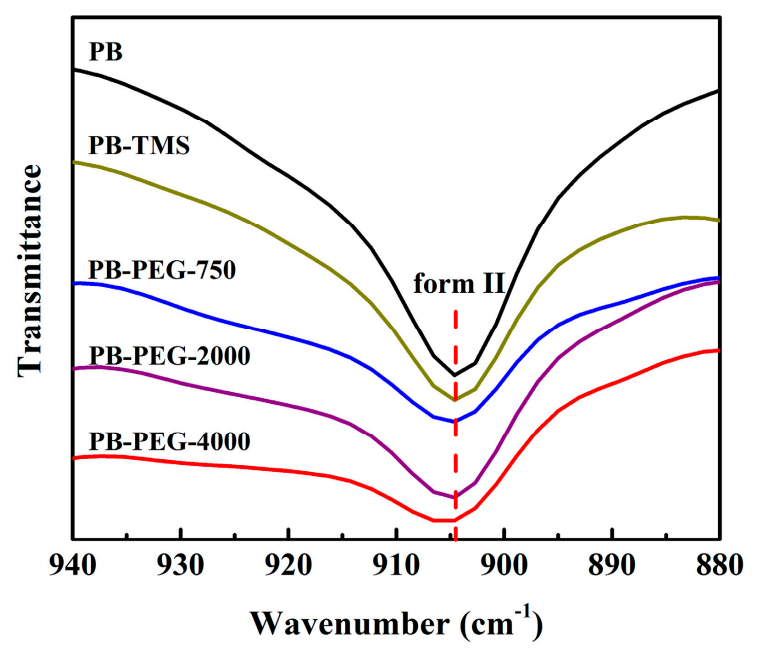

Figure 2. Fourier transform infrared spectroscopy (FTIR) spectrum of the PB, PB-TMS, and PB-PEG copolymers crystallized during cooling at $10^{\circ} \mathrm{C} / \mathrm{min}$.

Based on the observation that pure form II was generated, the degree of crystallinity $\left(X_{c}\right)$ is assessed using the following equation:

$$
X_{c}=\frac{\Delta H_{m}}{\Delta H_{I I}^{o}} \times 100 \%
$$

where $\Delta H_{m}$ is the melting enthalpy of crystallized form II and $\Delta H_{I I}^{o}$ is the melting enthalpy of the pure form II crystal (62 J/g) [49]. The determined values of $X_{\mathrm{c}}$ are shown in Table 2. Compared with $X_{\mathrm{c}}=$ $41 \%$ in $\mathrm{PB}$, incorporation of the TMS group decreases the crystallinity to $29.3 \%$. Interestingly, in the PB-PEG copolymers, the crystallinity was around 39\%, which is very close to linear PB. These results show the featured influence of PEG grafts on crystallization, which increase the cooling crystallization temperature but do not largely reduce the final degree of crystallinity.

\subsection{Isothermal Crystallization Kinetics}

In reality, crystallization can happen in a broader temperature range than the above cooling crystallization range. To understand the correlation between molecular factors and crystallization kinetics more comprehensively, isothermal crystallization was performed for the PB and PB-PEG copolymers at temperatures ranging from 50 to $85^{\circ} \mathrm{C}$. The crystallization kinetics were quantified by the normalized crystallinity index $\left(Z_{c}\right)$ using the following equation:

$$
Z_{c}(t)=\frac{\Delta H_{m}(t)}{\Delta H_{\infty}}
$$

where $\Delta H_{m}(t)$ is the integrated enthalpy over the total isothermal period of $t$ and $\Delta H_{\infty}$ is the enthalpy of the complete crystallization. Figure 3 displays the crystallization kinetics of the linear $\mathrm{PB}$ and grafted PB-PEG copolymers at three representative temperatures of 60,80 , and $85^{\circ} \mathrm{C}$ (the results of other temperatures are given in Figure S4). It was unexpected to observe that the PB and PB-PEG copolymers have different temperature dependences of crystallization kinetics. At the relatively low temperature of $60^{\circ} \mathrm{C}$, all PB-PEG copolymers have faster kinetics than PB, which is consistent with the increased $T_{\mathrm{C}}$ shown in Figure 1. At an elevated temperature of $80^{\circ} \mathrm{C}$, the crystallization of $\mathrm{PB}$ becomes faster than that of PB-PEG-2000 and PB-PEG-4000, but still slower than PB-PEG-750. As temperature was further increased to $85^{\circ} \mathrm{C}$, the crystallization kinetics of $\mathrm{PB}$ exceed all of the PB-PEG 
copolymers. The isothermal crystallization kinetics were analyzed using the Avrami model that can be described by the modified expression $\log \left[-\ln \left(1-Z_{c}(t)\right)\right]=\log k+n \log t$, with the exponent number $n$ and rate constant $k$. The insets of Figure 3 show the Avrami plots of crystallization in the homopolymer and PEG-grafted copolymers, in which the slope corresponds to the value of $n$. The quite close values of exponent number $n$ suggest that the introduction of long PEG grafts do not vary the crystallization dimensionality.

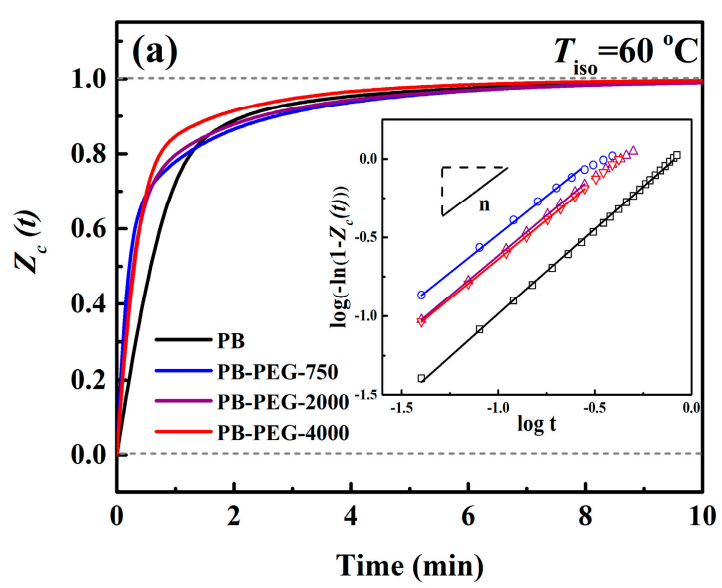

(a)

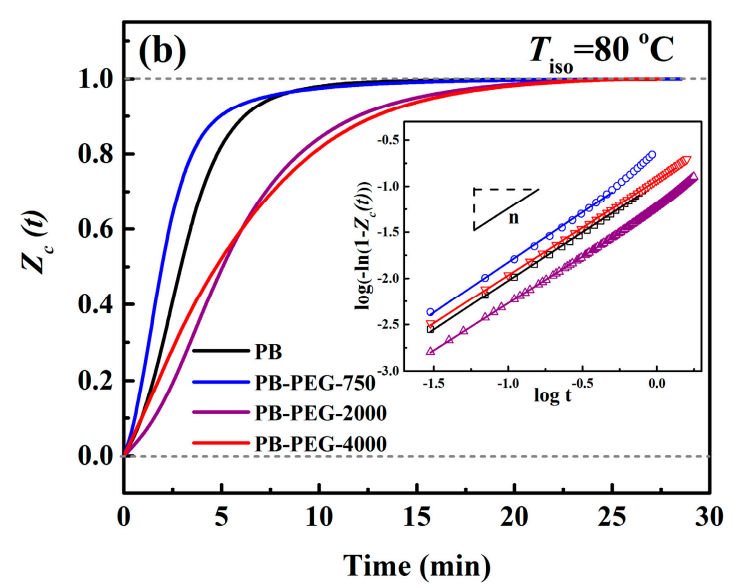

(b)

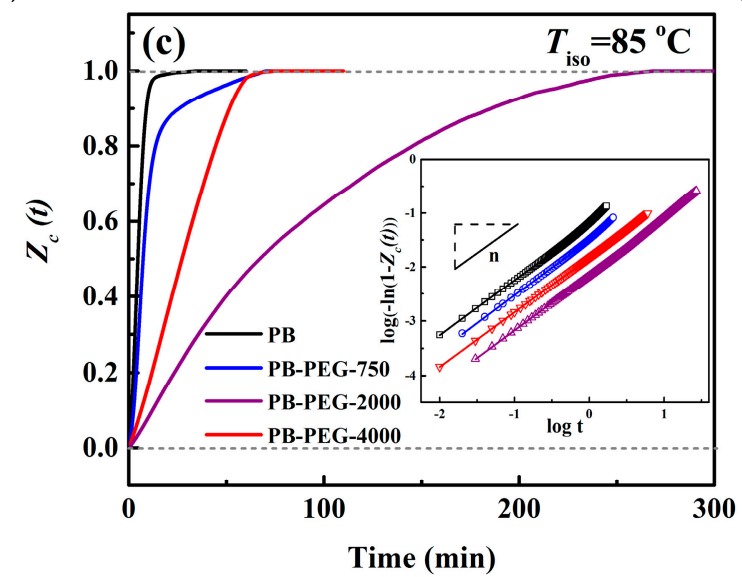

(c)

Figure 3. Kinetics of isothermal crystallization at temperatures of (a) 60 , (b) 80 , and (c) $85^{\circ} \mathrm{C}$.

Figure 4 shows the determined half-crystallization time $\left(t_{1 / 2}\right)$ for the PB and PB-PEG copolymers. Clearly, crystallization is slowed down with increasing temperature for all polymers. The resulting change of kinetics is more pronounced in the PB-PEG copolymers than in the linear PB. According to the relative sequence of crystallization kinetics, two temperature regions can be distinguished, where a threshold temperature is located at $70^{\circ} \mathrm{C}$. For the low temperature region $\left(T_{\text {iso }} \leq 70^{\circ} \mathrm{C}\right)$, incorporating PEG grafts to main chain accelerates crystallization with respect to linear PB. For example, at $50{ }^{\circ} \mathrm{C}$, reaching half crystallization requires around $20 \mathrm{~s}$ for $\mathrm{PB}$, which is almost twice that of the PB-PEG copolymers. Then, in the high temperature region $\left(T_{\text {iso }}>70^{\circ} \mathrm{C}\right)$, temperature elevation depresses PB-PEG crystallization more than PB. 


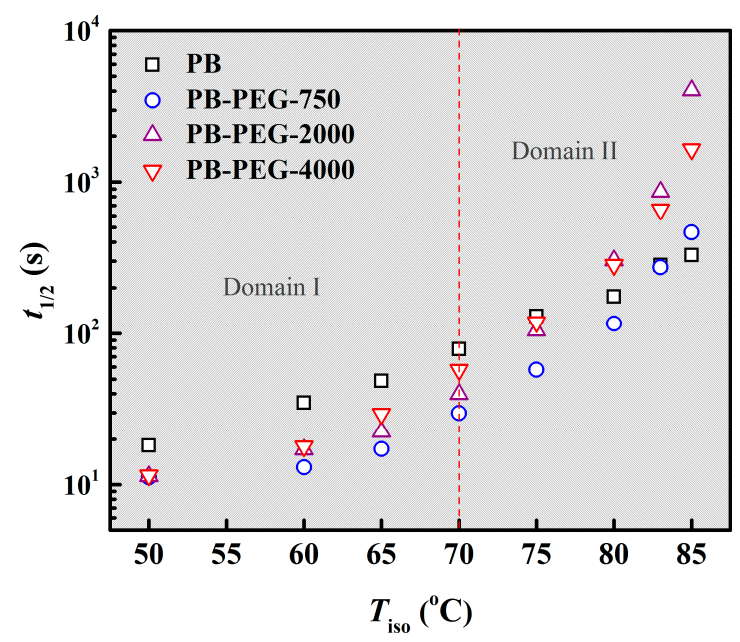

Figure 4. Half-crystallization time $\left(t_{1 / 2}\right)$ of PB and the PB-PEG copolymers as a function of isothermal crystallization temperatures.

It is known that crystallization is complemented by nucleation and growth steps. Figures S5 and $\mathrm{S} 6$ compare the nucleation densities after crystallization at $60^{\circ} \mathrm{C}$ and $85{ }^{\circ} \mathrm{C}$, respectively, which are representative of low and high temperature regions, respectively. As shown in Figure S5, the incorporation of a TMS branch is already able to increase the nucleation density significantly, while the presence of long PEG grafts enhances nucleation further. Considering the results in Figure 1, that PB-TMS crystallization capability is lower than PB, it could be inferred that the linear growth rate is smaller in PB-TMS than PB. This also implies that the growth rate is further reduced in PB-PEG copolymers, as observed in the long-chain branched PLA samples [41]. This implies that in PB-TMS and PB-PEG copolymers, nucleation density was increased but the linear growth rate was decreased with respect to PB. It should be noted that the POM results of Figures S5 and S6 cannot support the comparison among the different PEG graft lengths studied in this work. Then, whether increasing PEG graft length from 750 to $4000 \mathrm{~g} / \mathrm{mol}$ enhances nucleation density or not requires further investigation.

\subsection{Melting Behavior}

Figure 5 gives the DSC melting curves of the crystallites after isothermal crystallization at different temperatures, in order to examine the thermal stability of the crystallites. Although the crystallizations happened at identical temperatures, the melting temperatures of PB-PEG copolymers are all lower than that of PB crystals. Especially, PB-PEG form II crystallized at $85^{\circ} \mathrm{C}$ melts at $T_{\mathrm{m}}=104.9^{\circ} \mathrm{C}$, which is even lower than $T_{\mathrm{m}}=109.5^{\circ} \mathrm{C}$ of $\mathrm{PB}$ crystallized at $50^{\circ} \mathrm{C}$.

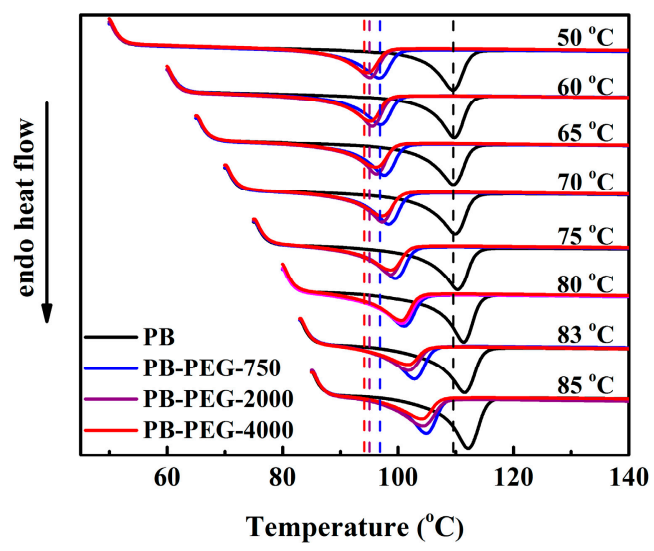

Figure 5. DSC melting curves of PB and PB-PEG crystallites prepared by isothermal crystallization at different temperatures. The thermal protocol was illustrated in Figure S3b. 
Figure 6 gives the melting temperatures $T_{\mathrm{m}}$ of PB and PB-PEG as a function of crystallization temperature $T_{\text {iso }}$. For all polymers, there is one critical crystallization temperature, below which the melting temperature is constant for different crystallization temperatures. This is associated to the recrystallization process that occurs during heating [50]. For PB, the critical crystallization temperature is $70^{\circ} \mathrm{C}$, which agrees well with the value reported by Wang et al. [51]. However, the introduction of PEG grafts decreases the critical temperature to $60^{\circ} \mathrm{C}$.

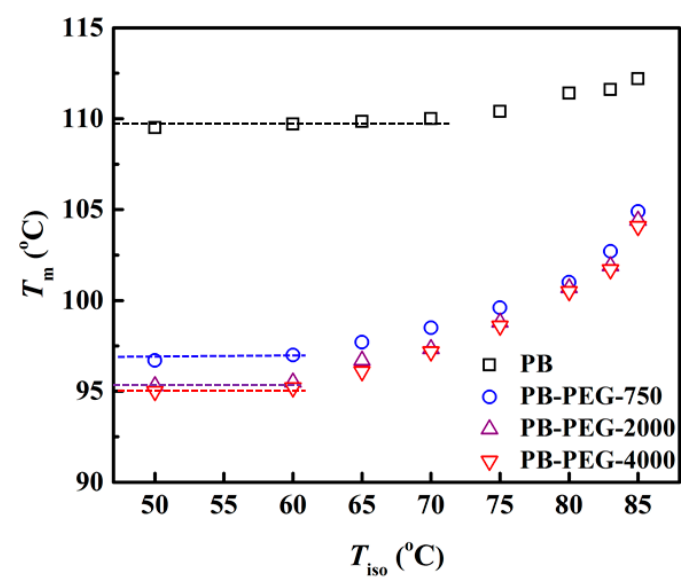

Figure 6. The melting temperatures of the PB and PEG-grafted polybutene-1 crystallites prepared by isothermal crystallization at different temperatures.

\subsection{Polymorphic Form II to Form I Phase Transition}

For polybutene- 1 homopolymer and copolymers, the melt-crystallized form II tends to transform into the stable form I. The crystallites generated by the above isothermal crystallization were annealed at $25^{\circ} \mathrm{C}$ for $50 \mathrm{~h}$ to allow for II-I phase transition. Without phase transition, the preparation of pure form II crystallites was also confirmed by the XRD measurements (Figure S7). Figure 7 shows the DSC melting curves of partially transformed samples for the crystallites prepared by isothermal crystallization at $60{ }^{\circ} \mathrm{C}$. As shown by the dashed lines, the initial form (form II) has a single endothermic peak. As soon as the II-I phase transition happens, extra melting peaks emerge at the high temperatures, which corresponds to the transformed form I [18,34]. It is clear that the II-I phase transition happens for all of the copolymers studied in this work. When the transition duration is fixed, the fraction of transformed form I corresponds to the transition rate. Fractions of transformed form I in total crystallites $\left(f_{\mathrm{I}}\right)$ are quantified using the following equation:

$$
f_{I}=\frac{\Delta H_{m_{-} I} / \Delta H_{I}^{0}}{\Delta H_{m_{-} I} / \Delta H_{I}^{0}+\Delta H_{m_{-} I I} / \Delta H_{I I}^{0}}
$$

where $\Delta H_{m_{-} I}$ and $\Delta H_{m_{-} I I}$ are the measured melting enthalpies of forms I and II, respectively (Figure S8). $\Delta H_{I}^{0}$ and $\Delta H_{I I}^{0}$ represent the ideal melting enthalpies of forms I and II, which are 141 and $62 \mathrm{~J} / \mathrm{g}$, respectively [49]. For $25^{\circ} \mathrm{C}$ and $50 \mathrm{~h}$, the determined fractions of transformed form I are also given in Figure 7. In PB, the fraction of form I transformed at $25^{\circ} \mathrm{C}$ for $50 \mathrm{~h}$ is 0.11 . It can be seen that the II-I transition rate of grafted PB-PEG copolymers strongly depends on the PEG length. PB-PEG-750 has the largest transition rate of 0.15 after annealing for $50 \mathrm{~h}$. When increasing the PEG length to $M_{\mathrm{n}}=$ 2000 and $4000 \mathrm{~g} / \mathrm{mol}$, the transition rate decreases to 0.12 and 0.05 , respectively. The extension of the PEG graft increases the steric hindrance and retards phase transition. 


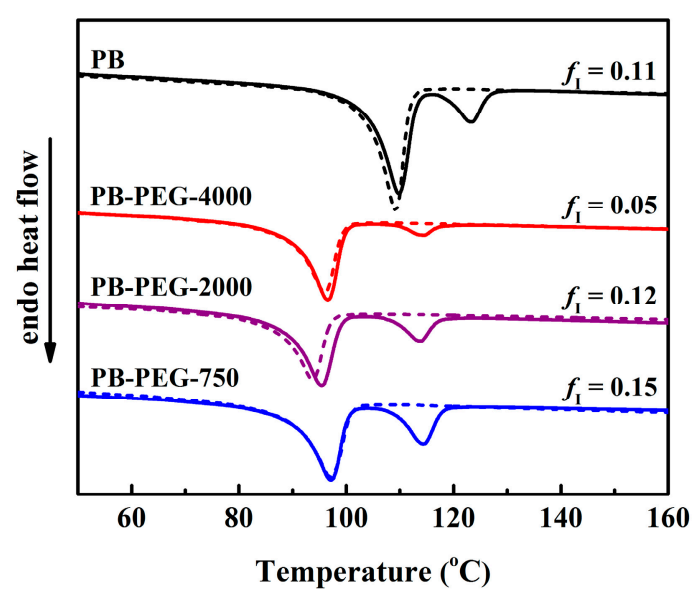

Figure 7. DSC melting curves of the PB and grafted PB-PEG copolymers after annealing at $25^{\circ} \mathrm{C}$ for 50 $\mathrm{h}$ (solid line), where initial form II was crystallized isothermally at $60^{\circ} \mathrm{C}$. The dashed line indicates the melting curves of form II without annealing.

Figure 8 summarizes the transition rates of $\mathrm{PB}$ and PEG-grafted copolymers for a broader crystallization temperature (DSC melting data given in Figure S9). Note that a very fast cooling rate of $50{ }^{\circ} \mathrm{C} / \mathrm{min}$ was utilized to prevent cooling crystallization and allow isothermal crystallization at a broad temperature range from 50 to $83^{\circ} \mathrm{C}$. It can be seen that the temperature dependence of the phase transition rate varies vastly with different lengths of PEG grafts. For linear PB, the transition rate often decreases with a decreasing crystallization temperature. This is due to the reason that when the transition temperature is fixed at $25^{\circ} \mathrm{C}$, the decrease of crystallization temperature reduces the internal thermal stress caused, which is the driving force for phase transition. Similarly, in PB-PEG copolymers with long grafts of $M_{\mathrm{n}}=2000$ and $4000 \mathrm{~g} / \mathrm{mol}$, transition rates decrease with reducing crystallization temperatures. As the PEG grafts were increased from $M_{n}=2000$ to 4000, the transition rate was reduced. This is probably because of the steric effect of the PEG grafts, which retards the necessary helical and positional adjustment of the crystalline stems within the crystals [15,31]. However, in PB-PEG-750, with the shortest PEG grafts, the transition rate increases with decreasing crystallization temperature, similar to the PB homopolymer with $M_{\mathrm{W}}=77 \mathrm{~kg} / \mathrm{mol}$ [33]. In summary, the II-I phase transition can be tuned by the control of the PEG length.

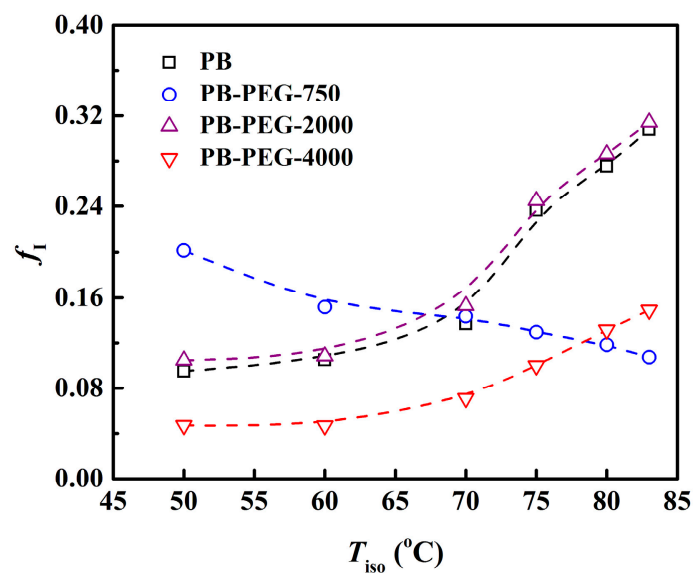

Figure 8. Fraction of transformed form I $\left(f_{\mathrm{I}}\right)$ in the PB and PEG-grafted copolymers as a function of isothermal crystallization temperature. The thermal protocol is illustrated in Figure S3c.

\section{Conclusions}

In this work, comonomer Ph-TMS was copolymerized with butene- 1 to obtain the trimethylsilyl and carbon-carbon triple bond groups, which can be employed to further prepare grafted butene- 1 
copolymers with different PEG grafts $\left(M_{\mathrm{n}}=750,2000\right.$ and $\left.4000 \mathrm{~g} / \mathrm{mol}\right)$. The crystallization, melting, and phase transition were systematically investigated. The DSC results showed that for cooling crystallization, the incorporation of a TMS branch significantly decreases the peak temperature of cooling crystallization, by more than $20^{\circ} \mathrm{C}$ with respect to the linear polybutene- 1 homopolymer. However, PEG-grafted copolymers have a greater crystallization temperature than PB-TMS, which is even higher than that of PB. This indicates that presence of PEG grafts enhances copolymer crystallization, although PEG grafts also disturb the regularity of main chains. Then, the results of isothermal crystallization demonstrate that there is a critical temperature of $70{ }^{\circ} \mathrm{C}$, below which the crystallization of PB-PEG copolymers is always faster than that of PB. In the temperature region above $70^{\circ} \mathrm{C}, \mathrm{PB}$ crystallization may exceed that of PB-PEG copolymers, depending on whether the increase of nucleation density can compensate the reduction of growth rate. Moreover, the DSC heating results display that the melting temperatures of the PB-PEG copolymer are lower than that of the PB.

When form II, crystallized at $60^{\circ} \mathrm{C}$, is annealed at $25^{\circ} \mathrm{C}$, PB-PEG-750, with $M_{\mathrm{n}}=750 \mathrm{~g} / \mathrm{mol}$ grafts, exhibits an accelerated transition rate that is faster than the homopolymer PB. However, a negative correlation between the transition rate and crystallization temperature was identified in PB-PEG-750 for a broad temperature range of $50^{\circ} \mathrm{C}$ to $83^{\circ} \mathrm{C}$. The transition rate of PB-PEG-2000 is very close to that of PB. As the PEG length was further increased to $4000 \mathrm{~g} / \mathrm{mol}$, the transition rate was retarded, which is probably due to steric effect.

Supplementary Materials: The following are available online at http://www.mdpi.com/2073-4360/11/5/837/s1. NMR results, GPC results, kinetics of isothermal crystallization at other temperatures, POM results, fitting method of DSC data, DSC melting curves of samples crystallized at different temperatures after annealing at $25^{\circ} \mathrm{C}$ for $50 \mathrm{~h}$.

Author Contributions: Conceptualization, C.A., Z.M. and Y.L. (Yuesheng Li); Data curation, C.A., Y.L. (Yahui Lou), B.W., L.P. and Z.M.; Formal analysis, C.A., Y.L. (Yahui Lou) and Z.M.; Investigation, C.A., Y.L. (Yahui Lou) and Z.M.; Resources, C.A. and Y.L. (Yulian Li); Writing—original draft, C.A.; Writing-review and editing, C.A., D.S. and Z.M.

Funding: This research was funded by the National Natural Science Foundation of China (No. 21690071 and 51873151). In addition, we also thank Yulan Chen and Miss Mengiiao Wu for the help with DSC experiments.

Conflicts of Interest: The authors declare no conflict of interest.

\section{References}

1. Nakafuku, C.; Miyaki, T. Effect of pressure on the melting and crystallization behaviour of isotactic polybutene-1. Polymer 1983, 24, 141-148. [CrossRef]

2. Luciani, L.; Seppälä, J.; Löfgren, B. Poly-1-butene: Its preparation, properties and challenges. Prog. Polym. Sci. 1988, 13, 37-62. [CrossRef]

3. Azzurri, F.; Flores, A.; Alfonso, G.C.; Baltá Calleja, F.J. Polymorphism of isotactic poly(1-butene) as revealed by microindentation hardness. 1. kinetics of the transformation. Macromolecules 2002, 35, 9069-9073. [CrossRef]

4. Azzurri, F.; Flores, A.; Alfonso, G.C.; Sics, I.; Hsiao, B.S.; Baltá Calleja, F.J. Polymorphism of isotactic polybutene- 1 as revealed by microindentation hardness. Part II: Correlations to microstructure. Polymer 2003, 44, 1641-1645. [CrossRef]

5. Lou, Y.H.; Liao, Y.L.; Pan, L.; Wang, B.; Li, Y.S.; Ma, Z. Effect of linear and ring-like co-units on the temperature dependence of nucleation and growth in II-I phase transition of butene-1 copolymers. Chin. J. Polym. Sci. 2018, 36, 1269-1276. [CrossRef]

6. Li, J.Q.; Wang, D.; Cai, X.Q.; Zhou, C.B.; de Claville Christiansen, J.; Sørensen, T.; Yu, D.H.; Xue, M.L.; Jiang, S.C. Conformation selected direct formation of form I in isotactic poly(butene-1). Cryst. Growth Des. 2018, 18, 2525-2537. [CrossRef]

7. De Rosa, C.; Auriemma, F.; Ruiz de Ballesteros, O.; Esposito, F.; Laguzza, D.; Di Girolamo, R.; Resconi, L. Crystallization properties and polymorphic behavior of isotactic poly(1-butene) from metallocene catalysts: The crystallization of form I from the melt. Macromolecules 2009, 42, 8286-8297. [CrossRef] 
8. Liu, Y.P.; Cui, K.P.; Tian, N.; Zhou, W.Q.; Meng, L.P.; Li, L.B.; Ma, Z.; Wang, X.L. Stretch-induced crystal-crystal transition of polybutene-1: An in situ synchrotron radiation wide-angle X-ray scattering study. Macromolecules 2012, 45, 2764-2772. [CrossRef]

9. Wang, Y.T.; Liu, P.R.; Lu, Y.; Men, Y.F. Mechanism of polymorph selection during crystallization of random butene-1/ethylene copolymer. Chin. J. Polym. Sci. 2016, 34, 1014-1020. [CrossRef]

10. Di Lorenzo, M.L.; Righetti, M.C. The three-phase structure of isotactic poly(1-butene). Polymer 2008, 49, 1323-1331. [CrossRef]

11. Xin, R.; Zhang, J.; Sun, X.1.; Li, H.H.; Ren, Z.J.; Yan, S.K. Polymorphic behavior and phase transition of poly(1-butene) and its copolymers. Polymers 2018, 10, 556. [CrossRef] [PubMed]

12. Qiao, Y.N.; Wang, H.; Men, Y.F. Retardance of form II to form I transition in polybutene-1 at late stage: A proposal of a new mechanism. Macromolecules 2018, 51, 2232-2239. [CrossRef]

13. Liu, Q.; Sun, X.L.; Li, H.H.; Yan, S.K. Orientation-induced crystallization of isotactic polypropylene. Polymer 2013, 54, 4404-4421. [CrossRef]

14. Zhang, B.; Yang, D.C.; Yan, S.K. Direct formation of form I poly(1-butene) single crystals from melt crystallization in ultrathin films. J. Polym. Sci. Part B Polym. Phys. 2002, 40, 2641-2645. [CrossRef]

15. Tashiro, K.; Hu, J.; Wang, H.; Hanesaka, M.; Saiani, A. Refinement of the crystal structures of forms I and II of isotactic polybutene- 1 and a proposal of phase transition mechanism between Them. Macromolecules 2016, 49, 1392-1404. [CrossRef]

16. Corradini, P.; Napolitano, R.; Petraccone, V.; Pirozzi, B. Conformational and packing energy for the three crystalline forms of isotactic poly- $\alpha$-butene. Eur. Polym. J. 1984, 20, 931-935. [CrossRef]

17. Danusso, F.; Gianotti, G. Isotactic polybutene-1: Formation and transformation of modification 2. Die Makromol. Chem. 1965, 88, 149-158. [CrossRef]

18. Qiao, Y.N.; Wang, Q.; Men, Y.F. Kinetics of nucleation and growth of form II to I polymorphic transition in polybutene-1 as revealed by stepwise annealing. Macromolecules 2016, 49, 5126-5136. [CrossRef]

19. Zheng, L.R.; Liu, L.; Shao, C.G.; Wang, W.; Wang, B.; Pan, L.; Li, Y.S.; Ma, Z. Phase transition from tetragonal form II to hexagonal form I of butene-1/4-methyl-1-pentene random copolymers: Molecular factor versus stretching stimuli. Macromolecules 2019, 52, 1188-1199. [CrossRef]

20. Tang, X.L.; Chen, W.; Li, L.B. The tough journey of polymer crystallization: battling with chain flexibility and connectivity. Macromolecules 2019. [CrossRef]

21. Androsch, R.; Hohlfeld, R.; Frank, W.; Nase, M.; Cavallo, D. Transition from two-stage to direct melt-crystallization in isotactic random butene-1/propene copolymers. Polymer 2013, 54, 2528-2534. [CrossRef]

22. Shi, J.Y.; Wu, P.Y.; Li, L.; Liu, T.; Zhao, L. Crystalline transformation of isotactic polybutene-1 in supercritical $\mathrm{CO}_{2}$ studied by in-situ fourier transform infrared spectroscopy. Polymer 2009, 50, 5598-5604. [CrossRef]

23. Wang, W.; Shao, C.G.; Zheng, L.R.; Wang, B.; Pan, L.; Ma, G.Q.; Li, Y.S.; Wang, Y.M.; Liu, C.T.; Ma, Z. Stretching-induced phase transition of the butene-1/ethylene random copolymer: Orientation and kinetics. J. Polym. Sci. Part B Polym. Phys. 2019, 57, 116-126. [CrossRef]

24. Wang, W.; Zheng, L.R.; Liu, L.Y.; Li, W.; Li, Y.S.; Ma, Z. Stretching behavior of the butene-1/ethylene random copolymer: A direct correspondence between triggering of II-I phase transition and mechanical yielding. Polym. Cryst. 2019, e10052. [CrossRef]

25. Hu, J.; Tashiro, K. Relation between higher-order structure and crystalline phase transition of oriented isotactic polybutene- 1 investigated by temperature-dependent time-resolved simultaneous WAXD/SAXS measurements. Polymer 2016, 90, 165-177. [CrossRef]

26. Zhang, X.X.; Li, Y.K.; Sun, Z.Y. Acceleration of crystal transformation from crystal form II to form I in Polybutene-1 induced by nanoparticles. Polymer 2018, 150, 119-129. [CrossRef]

27. Wanjale, S.D.; Jog, J.P. Poly(1-butene)/clay nanocomposites: Preparation and properties. J. Polym. Sci. Part B Polym. Phys. 2003, 41, 1014-1021. [CrossRef]

28. Tarallo, O.; Ruiz de Ballesteros, O.; Bellissimo, A.; Scoti, M.; Malafronte, A.; Auriemma, F.; De Rosa, C. Crystallization and mechanical properties of metallocene made 1-butene-pentene and 1-butene-hexene isotactic copolymers. Polymer 2018, 158, 231-242. [CrossRef] 
29. De Rosa, C.; Tarallo, O.; Auriemma, F.; Ruiz de Ballesteros, O.; Di Girolamo, R.; Malafronte, A. Crystallization behavior and mechanical properties of copolymers of isotactic poly(1-butene) with 1-octene from metallocene catalysts. Polymer 2015, 73, 156-169. [CrossRef]

30. Gianotti, G.; Capizzi, A. Butene-1/propylene copolymers. Influence of the comonomerie units on polymorphism. Die Makromol. Chem. 1969, 124, 152-159. [CrossRef]

31. Jones, A.T. Cocrystallization in copolymers of $\alpha$-olefins II-Butene- 1 copolymers and polybutene type II/I crystal phase transition. Polymer 1966, 7, 23-59. [CrossRef]

32. Azzurri, F.; Alfonso, G.C.; Gómez, M.A.; Martì, M.C.; Ellis, G.; Marco, C. Polymorphic transformation in isotactic 1-butene/ethylene copolymers. Macromolecules 2004, 37, 3755-3762. [CrossRef]

33. Qiao, Y.N.; Men, Y.F. Intercrystalline links determined kinetics of form II to I polymorphic transition in polybutene-1. Macromolecules 2017, 50, 5490-5497. [CrossRef]

34. Qiao, Y.N.; Yang, F.; Lu, Y.; Liu, P.R.; Li, Y.S.; Men, Y.F. Spontaneous form II to I transition in low molar mass polybutene-1 at crystallization temperature reveals stabilization role of intercrystalline links and entanglements for metastable form II crystals. Macromolecules 2018, 51, 8298-8305. [CrossRef]

35. Auriemma, F.; De Rosa, C.; Esposito, S.; Mitchell, G.R. Polymorphic superelasticity in semicrystalline polymers. Angew. Chem. Int. Ed. 2007, 46, 4325-4328. [CrossRef] [PubMed]

36. De Rosa, C.; Ruiz de Ballesteros, O.; Auriemma, F.; Di Girolamo, R.; Scarica, C.; Giusto, G.; Esposito, S.; Guidotti, S.; Camurati, I. Polymorphic behavior and mechanical properties of isotactic 1-butene-ethylene copolymers from metallocene catalysts. Macromolecules 2014, 47, 4317-4329. [CrossRef]

37. He, L.L.; Wang, B.; Yang, F.; Li, Y.S.; Ma, Z. Featured crystallization polymorphism and memory effect in novel butene-1/1,5-hexadiene copolymers synthesized by post-metallocene hafnium catalyst. Macromolecules 2016, 49, 6578-6589. [CrossRef]

38. Takamura, M.; Sugimoto, M.; Kawaguchi, S.; Takahashi, T.; Koyama, K. Influence of extrusion temperature on molecular architecture and crystallization behavior of peroxide-induced slightly crosslinked poly(L-lactide) by reactive extrusion. J. Appl. Polym. Sci. 2012, 123, 1468-1478. [CrossRef]

39. Wang, L.Y.; Jing, X.B.; Cheng, H.B.; Hu, X.L.; Yang, L.X.; Huang, Y.B. Rheology and crystallization of long-chain branched poly(l-lactide) s with controlled branch length. Ind. Eng. Chem. Res. 2012, 51, 10731-10741. [CrossRef]

40. Fang, H.G.; Zhang, Y.Q.; Bai, J.; Wang, Z.G. Shear-induced nucleation and morphological evolution for bimodal long chain branched polylactide. Macromolecules 2013, 46, 6555-6565. [CrossRef]

41. Bai, J.; Fang, H.G.; Zhang, Y.Q.; Wang, Z.G. Studies on crystallization kinetics of bimodal long chain branched polylactides. Cryst. Eng. Comm. 2014, 16, 2452. [CrossRef]

42. Wang, J.; Bai, J.; Zhang, Y.; Fang, H.; Wang, Z. Shear-induced enhancements of crystallization kinetics and morphological transformation for long chain branched polylactides with different branching degrees. Sci. Rep. 2016, 6, 26560. [CrossRef]

43. Hong, M.; Liu, J.Y.; Li, B.X.; Li, Y.S. Facile functionalization of polyethylene via click chemistry. Macromolecules 2011, 44, 5659-5665. [CrossRef]

44. Gao, H.F.; Matyjaszewski, K. Synthesis of molecular brushes by "grafting onto" method: Combination of ATRP and click reactions. J. Am. Chem. Soc. 2007, 129, 6633-6639. [CrossRef]

45. Ewen, J.A.; Elder, M.J.; Jones, R.L.; Rheingold, A.L.; Liable-Sands, L.M.; Sommer, R.D. Chiral ansa metallocenes with $\mathrm{Cp}$ ring-fused to thiophenes and pyrroles: Syntheses, crystal structures, and isotactic polypropylene Catalysts. J. Am. Chem. Soc. 2001, 123, 4763-4773. [CrossRef]

46. Wang, Z.F.; Dong, X.; Cavallo, D.; Müller, A.J.; Wang, D.J. Promotion of self-nucleation with latent form I nuclei in polybutene-1 and its copolymer. Macromolecules 2018, 51, 6037-6046. [CrossRef]

47. Yamashita, M. Direct crystal growth of isotactic polybutene-1 trigonal phase in the melt: In-situ observation. J. Cryst. Growth 2008, 310, 1739-1743. [CrossRef]

48. Li, L.; Liu, T.; Zhao, L.; Yuan, W.K. $\mathrm{CO}_{2}$-induced phase transition of isotactic poly-1-butene with form III upon heating. Macromolecules 2011, 44, 4836-4844. [CrossRef]

49. Rubin, I.D. Relative stabilities of polymorphs of polybutene-1 obtained from the melt. J. Polym. Sci. Part B Polym. Lett. 1964, 2, 747-749. [CrossRef] 
50. Heck, B.; Siegenführ, S.; Strobl, G.; Thomann, R. A law controlling polymer recrystallization showing up in experiments on s-polypropylene. Polymer 2007, 48, 1352-1359. [CrossRef]

51. Wang, Y.T.; Lu, Y.; Jiang, Z.Y.; Men, Y.F. Molecular weight dependency of crystallization line, recrystallization line, and melting line of polybutene-1. Macromolecules 2014, 47, 6401-6407. [CrossRef]

(C) 2019 by the authors. Licensee MDPI, Basel, Switzerland. This article is an open access article distributed under the terms and conditions of the Creative Commons Attribution (CC BY) license (http://creativecommons.org/licenses/by/4.0/). 